\title{
Adaptación en adolescentes que practican actividad física frente a los que no practican²
}

\author{
César Augusto Cetina Briceño \\ Eps. Psicología Clínica de la Niñez y la Adolescencia \\ Universidad de la Sabana \\ Chía, Colombia \\ Correo electrónico: psicologosocial@gmail.com
}

Jaime Humberto Moreno Méndez

$\mathrm{PhD}$, Psicología Industrial Organizacional Pontificia Universidad Católica de Puerto Rico Recinto de Ponce, Puerto Rico

Correo electrónico: jaimehm@yahoo.com

Recibido: 05/01/2015

Evaluado: 01/05/2015

Aceptado: 26/05/2015

\section{Resumen}

Objetivo: El propósito de la investigación fue describir y comparar el nivel de adaptación personal, escolar, familiar y social en adolescentes que practican actividad física frente a los que no practican. Método: Es un estudio descriptivo de tipo comparativo. Se aplicó el Test Autoevaluativo Multifactorial de Adaptación Infantil (TAMAl) de Hernández (1998) y el Cuestionario Internacional de Actividad Física (IPAQ) de Booth (2000) a 68 jóvenes de la ciudad de Duitama (Colombia) 34 por cada caracterización de grupo. Resultados: Los resultados mostraron que hubo diferencias significativas entre los adolescentes que practican y no practican actividad física siendo mayor la inadaptación escolar y social entre los que no practican. Conclusiones: las conclusiones conducen a que la adpatación del adolescente debe ser holística, comprendiendo varias áreas del desarrollo para que el ajuste sea coherente con las demandas ambientales. 


\title{
Adaptation in adolescents that practice physical activity compared to those who do not.
}

\begin{abstract}
Objective: the purpose of this study was to describe and compare the level of personal, school, family and social adjustment in adolescents who practice physical activity compared to those who do not practice it. Method: a descriptive study of comparative type. The Children Adaptation Multifactor Self-assessment Test (TAMAI for its initials in Spanish) by Hernández (1998) and the International Physical Activity Questionnaire (IPAQ) by Booth (2000) were applied to 68 young people from the city of Duitama (Colombia), 34 for each characterization group were applied. Results: the results showed that there were significant differences between adolescents who practice physical activity and those who do not, resulting in larger school and social maladjustment among those who do not practice it. Conclusions: the conclusions lead to acknowledging that the adaptation of the teenager should be holistic, comprising several areas of development for the adjustment to be consistent with environmental demands.
\end{abstract}

\section{Adaptação em adolescentes que praticam atividade física perante a aqueles que não o fazem}

Resumo

Objetivo: o propósito da pesquisa foi fazer uma descrição e comparar o nível de adaptação pessoal, escolar, familiar e social dos adolescentes que praticam alguma atividade física perante aqueles que não o fazem. Método: trata-se dum estúdio descritivo de tipo comparativo. Aplicou-se o teste Autoevaluativo Multifuncinal de Adaptacao Infantil (TAMAI) de Hernandez (1988) e o questionário internacional de atividade física (IPAQ) de Booth (2000) a 68 jovens da cidade de Duitama (Colômbia), 34 por cada caracterização de grupo. Resultados: os resultados mostraram que houve algumas diferencias significativas entre os adolescentes que praticam esportes e os que não praticam atividade física, tendo como maior o desajustamento escolar e social entre os estudantes que não praticam. Conclusões: as conclusões conduzem a elucidar que a adaptação do adolescente deve ser holística, abarcando as varias áreas do desenvolvimento com 0 ajustamento seja coerente com as demandas do ambiente. 


\section{ntroducción}

El presente artículo es el resultado de la investigación desarrollada por Cetina y Moreno (2013) dentro de la Especialización en Psicología Clínica de la Niñez y la Adolescencia. Se encuentra vinculado dentro del Grupo de investigación Procesos Psicológicos y Contexto Social en la Línea de Procesos emocionales, conductuales y Bienestar Psicológico de la Facultad de Psicología de la Universidad de La Sabana. El estudio se llevó a cabo con adolescentes del SENA de la ciudad de Duitama, con el fin de profundizar en su nivel de ajuste desde perspectivas contemporáneas del desarrollo. Se aporta una perspectiva de la adaptación de los adolescentes estableciendo sus niveles de ajuste a nivel personal, social, escolar y familiar, teniendo en cuenta los resultados de cada adolescente y lo que les proporciona el ambiente. Se asume que la adaptación en la adolescencia comprende un proceso dinámico, en dónde los comportamientos se basan en preferencias relacionadas con el entorno en el que se desenvuelven, especialmente, con personas que son significativas.

La práctica de actividad física provee múltiples efectos favorables en los adolescentes, tales como el fortalecimiento de la autoestima y el mantenimiento de un estado de salud vital. Otros de los beneficios que promueven son el afianzamiento de las relaciones sociales en su grupo de pares, disminuyendo la probabilidad que sea participe de situaciones de riesgo, y se mantenga una conducta que permita su adaptación, por medio del acompañamiento de personas significativas de su entorno como padres, amigos o docentes (Gálvez, Rodríguez, \& Velandrino, 2007).

Rodrigo, Máiquez, García, Mendoza, Rubio, Martínez y Martín (2004) describen como el adolescente podría verse involucrado en actividades relacionadas con riesgo para su integridad, debido a que surge una necesidad de buscar nuevas experiencias para recibir la aceptación y respeto por parte de sus pares.

Para Lillo (2004) durante la adolescencia como ciclo, se elabora la identidad del sujeto que será base para la adultez. Es el momento en el que experimentar nuevas tendencias y estilos de vida posibilitan la construcción de un pensamiento a través de dichas vivencias. Tapia, Castro y Monestel (2007), destacan que en la adolescencia el desarrollo del pensamiento lógico y los sentimientos de independencia se caracterizan de igual forma en los dos sexos, con prevalencia de conductas prosociales.

Durante su proceso de adaptación, el adolescente refleja características relacionadas con el sexo. Rodrigo et al., (2004) identificaron como en la adolescencia media, las chicas no se sienten satisfechas con su cuerpo y no presentan regularidad en su alimentación; mientras que los chicos presentan menor rendimiento académico. Lo anterior debido a que su desarrollo neurológico lo determina el sexo, manifestándose comportamientos que podrían perjudicar su integridad, como la reducción en la ingesta de alimentos, y en donde su autorregulación les permite comportarse de acuerdo a su pensamiento lógico y a su cuestionamiento de normas. 
En lo que respecta a características de desarrollo, Gómez (2007) identificó a través de un estudio comparativo entre sexos en adolescentes, que las jóvenes mayores experimentaron sentimientos negativos como desinterés, aburrimiento tristeza y letargo; en lo que respecta al canal cognitivo, pensamientos catastróficos, absolutista y autoderrotista; situación contraria a la que vivieron los adolescentes (hombres), en los cuales se evidenciaron pensamientos de satisfacción, gusto, entusiasmo, unión, afirmación y confianza.

Rodrigo et al., (2004) hallaron relación entre las actividades de ocio y la adaptación escolar, describiendo que dichas actividades se centraban en el consumo de productos adictivos, directamente relacionado con una insatisfacción escolar, situaciones de repetición de curso o absentismo escolar. La existencia de ambientes socializadores con presencia de eventos esporádicos como agresiones, hacen que el adolescente se ajuste y se sienta en un estado de bienestar biospsicosocial disminuyendo estímulos discriminativos que incrementen comportamientos perturbadores, o signos clínicos patológicos; además se encontraron diferencias significativas en el rendimiento académico entre las y los adolescentes, evidenciando mayor adaptación por parte de las adolescentes debido a procesos como expectativas de seguir estudiando y sentido de pertenencia a la escuela, relaciones funcionales con el profesorado.

En cuanto a la familia y la adaptación escolar, Martínez, Musitu, Amador y Monreal (2012) hallaron que de acuerdo al apoyo que reciba el adolescente en su etapa escolar tendrá relación directa con la consecución de logros a largo plazo, el refuerzo sobre resultados académicos, actividades extraacadémicas, acompañamiento a programaciones académicas y recreativas, las cuales están relacionadas tanto con la autoestima como con la confianza que tenga el adolescente en su núcleo.

De la Fuente, Peralta y Sánchez (2009) hallaron como la autorregulación de los adolescentes contribuye con la convivencia escolar y reduciendo situaciones académicas desadaptativas como el acoso escolar. Para que se desarrolle un proceso de adaptación en la escuela, Díaz (2005), expone que las conductas prosociales permiten el ajuste del adolescente, enfatizando que dichos comportamientos deben ser entre los mismos adolescentes y con sus docentes, lo que generaría confianza en las relaciones sociales del adolescente en el contexto escolar, además de prevenir situaciones de violencia. Por su parte, Tapia et al., (2007) muestran como esas conductas prosociales basadas en la empatía, se basan teniendo en cuenta la perspectiva de la otra persona, así como la reciprocidad de las relaciones interpersonales.

La familia del adolescente es el recurso afectivo que contribuye al proceso de ajuste del sujeto. Martínez et al., (2012) y González y Otero (2005) identificaron que de acuerdo al nivel educativo de los padres, había una relación directa con la práctica de actividades saludables en los adolescentes, así como el apoyo por parte de los padres tiene diferenciación de acuerdo al sexo, puesto que cuando el adolescente percibe apoyo por parte de su padre, se asocia con una menor participación en conductas agresivas. 
La práctica de la actividad física conduce a la prevención de asumir conductas de riesgo y a fortalecer el apoyo de los cuidadores de los adolescentes, se contemplaría como una alternativa para mejorar la calidad de vida en el adolescente, reduciendo situaciones que puedan afectar su integridad tales como dificultades de salud (Varela, Duarte, Salazar, Lema, \& Tamayo, 2011), abuso de drogas y alcohol (Avellaneda, Gras, \& Font, 2010), problemas relacionados con la obesidad (Almeida, Salgado, \& Nogueira, 2011; Giraldo, Poveda, Forero, Mendivil, \& Castro 2008), además de tener concordancia entre dicha enfermedad y hábitos de sueño inadecuados para la edad; conductualmente, Carrasco, Gómez y Staforelli (2009) hallaron presencia de signos de ansiedad, depresión, agresión y sentimientos de exclusión en adolescentes que presentaban prácticas de actividades físicas que requieran un mínimo de esfuerzo o de dedicación, además de estar directamente relacionada la adaptación en altos niveles con la salud física (Urdampilleta, González, Infante, \& Goñi, 2011), así como también está asociado con la amplificación de respuestas de miedo e induciendo síntomas fisiológicos como temblor, fatiga, taquicardia, sudor (Molina, Sandín, \& Chorot, 2014).

Oliva (2007), afirma como comportamentalmente el adolescente practica actividades que podrían afectar su integridad, proponiendo como la puesta en marcha de actividades que mejoren su calidad de vida, como la realización de actividades físicas, podrían reducir acciones de riesgo como el abuso en el consumo de drogas. La actividad física produce un estado de bienestar biopsicosocial y es un reforzador de acuerdo tanto a las motivaciones como a las recompensas que ciertas características determinan el comportamiento del adolescente a la hora de practicar actividades físicas; Gálvez, Rodríguez y Velandrino (2007) hallaron en su investigación que el resultado más relevante en la práctica física era la interacción social, especificando el valor de las experiencias socio-afectivas con su grupo de pares.

Por otra parte, Martínez et al., (2012) no encontraron diferencias significativas entre mantener una actividad física y las condiciones socio-económicas, dado que la limitación de recursos económicos no es equivalente a no practicar actividad física; pero sí hacen evidente la diferenciación entre la adolescencia temprana y media, dado que a más temprana edad, más probabilidades hay que el sujeto mantenga esta conducta durante otras etapas, situación similar sucede desde la Infancia. Algunos autores (Ángeles, 2011; Gálvez et al., 2007) concluyen que para reducción de riesgos en los adolescentes y la prevención de presencia de problemas psicológicos, la implementación de programas colaborativos comunitarios de carácter extra escolar aumentan procesos de empatía, pensamiento lógico, elevan el autoestima y potencian una vocación para la vida adulta.

Con base en el desarrollo cotidiano de integridad del Adolescente en lo académico, una variable directa con su desempeño y con su funcionalidad es la nutrición, el consumo de alimentos saludables, los cuales contribuyen al desarrollo de un estado de bienestar y a un ajuste del adolescente en este 
contexto especifico (Pastor, Balaguer, \& García, 2005). Adicionalmente se analiza que desde la prevención de conductas de riesgo, los contextos escolares y extraescolares tienen la oportunidad de brindar espacios y programas para mejorar condiciones de calidad de vida de los adolescentes; idea análoga a la práctica de actividad física.

Siendo la práctica de actividad física contribuyente a un estado psicosocial funcional del adolescente y su proceso de adaptación, Seder, Villalonga y Domingo (2014) describen como a través de dicha práctica se potencia la planificación de tiempo y la realización de actividades tanto académicas como familiares, siendo más utilizadas estas herramientas en las adolescentes mujeres.

Adaptarse en la adolescencia es la dinámica entre la maduración cerebral, los comportamientos manifiestos y la sociedad. La conducta del adolescente no sólo parte de un principio de satisfacción, sino de un conglomerado emocional que va asumiendo su personalidad y adquisición de herramientas psicológicas que le permitan una funcionalidad familiar y con sus pares que conlleven al cumplimiento de sus propósitos académicos, de salud y de convivencia; es así como surge la pregunta ¿Cómo se manifiesta la adaptación en adolescentes que practican y que no practican actividad física?. El objetivo principal es identificar y comprar el nivel de adaptación personal, escolar, familiar y social de adolescentes que practican y que no practican actividad física en el Servicio Nacional de Aprendizaje (SENA) de la cuidad de Duitama

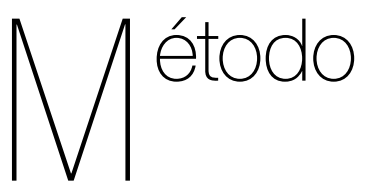

\section{Diseño}

El presente estudio investigativo es de corte empírico-analítico, con un diseño descriptivo de tipo comparativo y transeccional. Los diseños de investigación transeccional o transversal recolectan datos en un solo momento, en un tiempo único. Su propósito es describir variables y analizar su prevalencia e interrelación en un momento dado (Hernández, Fernández, \& Baptista, 2010). Para la presente investigación se describieron y compararon los niveles de adaptación personal, familiar, educativa y social de los estudiantes del SENA que practican o no actividad física.

\section{Participantes}

Para el presente Estudio participaron 68 adolescentes pertenecientes al SENA de la ciudad de Duitama. El universo fue de 185 estudiantes del SENA, de los cuales 137 eran adolescentes. Se solicitó autorización a padres de familia, aprobando en su totalidad el desarrollo de la investigación y la aplicación de los instrumentos. Los adolescentes que decidieron participar fueron 86 a través del asentimiento informado; posteriormente se les aplicó el Cuestionario Internacional de Actividad Física (IPAQ) para seleccionar los que no practican y practican actividad física, arrojando que 34 no practican actividad física y se tomó el mismo número aleatorio de los que practican actividad física. 
Para efectos del estudio se utiliza muestreo no probabilístico de sujetos tipo, consistente en la profundidad y en la cantidad de información, analizando el significado de uno o varios grupos en particular (Hernández, Fernández, \& Baptista, 2010).

En cuanto a la descripción de la población, con respecto al sexo, la participación de la Mujer fue de un 59\%, frente a la del Hombre con un 41\%; las mujeres que Practican Actividad Física y las que No Practican tienen un $71 \%$, de la misma forma, los hombres tanto los que Practican como los que No Practican tienen un 29\%; de la población total del estudio, el 59\% cuentan con 16 años de edad, el $32 \%$ cuenta con 17 años y el 8\% cuenta con 18 años; el estrato con mayor prevalencia es el 2 con un $78 \%$, estrato 3 con un $13 \%$ y estrato 4 con un 9\%. Los Técnicos y Tecnólogos que cursan los Adolescentes están relacionados con temas Agropecuarios y Agroindustriales.

\section{Instrumentos}

Cuestionario Internacional de Actividad Física (IPAQ) (Booth, 2000): El cuestionario clasifica la actividad física en tres niveles: baja, moderada y alta. En la categoría alta se incluyen sujetos muy activos; en tanto que en la baja, sujetos sedentarios. Para la actual investigación, se seleccionaron los sujetos que no practican actividad física con la calificación de bajo, mientras que para los adolescentes que practican actividad física, las calificaciones de medio y alto, teniendo como criterio la puntuación categórica del cuestionario y tiempo de dedicación, versión corta (Varela et al., 2011). Su fiabilidad test-retest es de rs $1 / 4$ .8-.96, con una validez de rs $1 / 4.33$ y de r 1/4 .67. Se expresan en METs minutos/ semana. El IPAQ considera actividades de intensidad moderada a aquellas de 3-6 METs y vigorosas a aquellas mayores de 6 METs y emplea los siguientes equivalentes metabólicos para cada nivel de actividad: caminar $=3.3$; actividad moderada $=4.0$; actividad vigorosa $=8.0$. Así, los METs min/semana corresponden al equivalente metabólico $\times$ minutos al día $\times$ días a la semana (Parada, Klaasen, Lisboa, Saldías, Mendoza, \& Díaz, 2011).

Test Autoevaluativo Multifactorial de Adaptación Infantil TAMAl (Hernández, 1998): Es una prueba autoevaluativa que consta de 175 ítems, que se responde afirmativa o negativamente con el propósito de identificar niveles de adaptación personal, familiar, social y escolar. Su aplicación de forma individual. El test evalúa la inadaptación personal, que comprende desde el desajuste del adolescente que tiene consigo mismo, como con su cotidianidad; la inadaptación social, como el significado del grado de incapacidad o de dificultades que podrían surgir en la relación social; la inadaptación escolar, que evalúa la insatisfacción y comportamientos disfuncionales respecto a la realidad escolar; la insatisfacción con la familia, relacionado con el ambiente psicosocial del hogar y la relación de los cuidadores entre sí; la insatisfacción con los hermanos, que analiza las diferencias y una relación negativa entre los hermanos y, finalmente, las pautas de crianza de los padres, donde los estilos educativos de los padres son evaluados según la percepción de los adolescentes. En cuanto a su fiabilidad, se ha utilizado el procedimiento de las dos mitades con la corrección de la fórmula de Sperman-Brown, obteniendo un índice de fiabilidad del .87, para el test en general. En relación con los tres niveles estudiados, los 
resultados son como siguen: Primer Nivel .67; Segundo Nivel .89 y Tercer Nivel .83.

El nivel 3 del TAMAl comprende de los 14 años y 6 meses a los 18 años y se califica: MB (Muy Bajo; centil 1 a 5); B (Bajo; centil de 6 a 20); CB (Casi Bajo; centil 21 a 40); M (Medio; centil 41 a 60); CA (Casi Alto; centil 61 a 80); A (Alto; centil 81 a 95); MA (Muy Alto; centil 96 a 99); para el presente estudio, desde el centil 61 se consideró como inadaptación.

\section{Procedimiento}

Fase 1: Se explicaron los objetivos de la investigación a las directivas del SENA de la ciudad de Duitama, con el propósito de obtener la autorización para el desarrollo de la investigación.

Fase 2: Explicación de la investigación a Padres de familia y a los adolescentes para la obtención de los consentimientos y asentimientos informados, así como el propósito del cuestionario IPAQ y del instrumento TAMAl; además de la recolección de datos frente a la caracterización de los sujetos de la investigación

Fase 3: Análisis de resultados y discusión, los cuales fueron socializados a las directivas y demás interesados del SENA de la ciudad de Duitama.

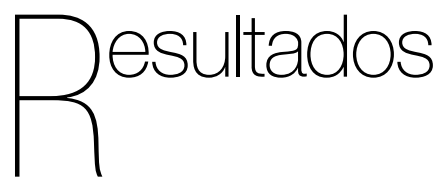

En primera instancia se encontrará el análisis estadístico de prueba $T$ para muestras relacionadas distribuidas de acuerdo a las subescalas del TAMAI y por característica de los sujetos; y por último, un análisis de frecuencias y porcentajes de las respuestas que se presentaron en el estudio. Para efectos del análisis estadístico descriptivo, la variable adaptación también fue medida de forma ordinal de acuerdo a las categorías ofrecidas por el instrumento: análisis de frecuencias. Los resultados comparativos se presentan de acuerdo a cada subescala del TAMAl, así como por la comparación de los grupos que practican actividad física y no practican actividad física.

Como se aprecia en la Tabla 1, se encontraron diferencias estadísticamente significativas entre los grupos que practican actividad física en relación con los que no practican actividad física, con una puntuación de .026, lo que significa que la práctica reducida de la actividad física tiene relación directa con la inadaptación escolar.

Tabla 1

Estadístico Comparativo Subescala TAMAI Inadaptación Escolar entre los grupos del estudio

\begin{tabular}{|c|c|c|c|c|c|c|c|c|c|}
\hline & & \multicolumn{5}{|c|}{ Diferencias relacionadas } & \multirow{3}{*}{$\mathrm{t}$} & \multirow{3}{*}{ gl } & \multirow{3}{*}{$\begin{array}{c}\text { Sig. } \\
\text { (bilateral) }\end{array}$} \\
\hline & & \multirow[t]{2}{*}{ Media } & \multirow[t]{2}{*}{ Desv. Típ. } & \multirow{2}{*}{$\begin{array}{l}\text { Error } \\
\text { típ. de la } \\
\text { media }\end{array}$} & \multicolumn{2}{|c|}{$\begin{array}{l}95 \% \text { Intervalo de } \\
\text { confianza para } \\
\text { la diferencia }\end{array}$} & & & \\
\hline & & & & & Inferior & Superior & & & \\
\hline Par 1 & $\begin{array}{l}\text { Inadaptacion.Escolar.No.Act.Fisica } \\
\text { - Inadaptacion.Escolar.Act.Fisica }\end{array}$ & 15.588 & 39.118 & 6.709 & 1.939 & 29.237 & 2.324 & 33 & .026 \\
\hline
\end{tabular}


Según la Tabla 2, hay diferencias estadísticamente significativas entre los grupos que practican actividad física con relación a los que no practican actividad física, presentándose mayor inadaptación social, con una puntuación de 0,023, siendo significativo el apoyo por parte del grupo de pares en la adolescencia; el cual refuerza y/o mantiene la conducta de la práctica de actividad, así como genera un estado emocional adaptativo, en contraste con los que no practican.

Tabla 2

Estadístico Comparativo Subescala TAMAl Inadaptación Social entre los grupos del estudio

\begin{tabular}{|c|c|c|c|c|c|c|c|c|c|}
\hline & & \multicolumn{5}{|c|}{ Diferencias relacionadas } & \multirow{3}{*}{$\mathrm{t}$} & \multirow{3}{*}{ gl } & \multirow{3}{*}{$\begin{array}{c}\text { Sig. } \\
\text { (bilateral) }\end{array}$} \\
\hline & & \multirow[t]{2}{*}{ Media } & \multirow{2}{*}{$\begin{array}{l}\text { Desviación } \\
\text { típ. }\end{array}$} & \multirow{2}{*}{$\begin{array}{c}\text { Error } \\
\text { típ. de } \\
\text { la media }\end{array}$} & \multicolumn{2}{|c|}{$\begin{array}{l}95 \% \text { Intervalo de } \\
\text { confianza para } \\
\text { la diferencia }\end{array}$} & & & \\
\hline & & & & & Inferior & Superior & & & \\
\hline Par 1 & $\begin{array}{l}\text { Inadaptacion.Social.No.Act.Fisica } \\
\text { - Inadaptacion.Social.Act.Fisica }\end{array}$ & 11.500 & 28.165 & 4.830 & 1.673 & 21.327 & 2.381 & 33 & .023 \\
\hline
\end{tabular}

No se encontraron diferencias estadísticamente significativas en la inadaptación personal y en la insatisfacción familiar, entre los grupos que practican actividad física en comparación con los que no practican actividad física de acuerdo a la aplicación de la Prueba $T$ para muestras relacionadas, con una puntuación de .299 y de .418 respectivamente.
Con base en la Tabla 3, se identifica mayor nivel de inadaptación personal en los que no practican actividad física en la puntuación Alta (35.3\%), seguido por las calificaciones de Casi Alto y Casi Bajo con un $20.6 \%$ respectivamente; la categoría Media con un 2.9\% en la subescala. En los que practican actividad física, se evidencia que en nivel Muy Alto el porcentaje es un $23.5 \%$, siendo la mayor frecuencia en esta muestra.

Tabla 3

Frecuencias y Porcentajes de la Subescala TAMAl en los grupos de estudioen Inadaptación Personal

\begin{tabular}{|c|c|c|c|c|c|c|c|}
\hline $\begin{array}{l}\text { Inadaptación } \\
\text { Personal No } \\
\text { Actividad } \\
\text { Física }\end{array}$ & Categoría & Frecuencia & $\%$ & $\begin{array}{l}\text { Inadaptación } \\
\text { Personal } \\
\text { Actividad } \\
\text { Física }\end{array}$ & Categoría & Frecuencia & $\%$ \\
\hline & Muy bajo & 5 & 14.7 & & Muy bajo & 5 & 14.7 \\
\hline & Bajo & 2 & 5.9 & & Bajo & 6 & 17.6 \\
\hline & Casi Bajo & 7 & 20.6 & & Casi Bajo & 6 & 17.6 \\
\hline & Medio & 1 & 29 & & Medio & 2 & 5.9 \\
\hline & Casi Alto & 7 & 20.6 & & Casi Alto & 6 & 17.6 \\
\hline & Alto & 0 & 35.3 & & Alto & 1 & 2.9 \\
\hline & Muy Alto & 12 & 0 & & Muy Alto & 8 & 23.5 \\
\hline & Total & 34 & 100 & & Total & 34 & 100 \\
\hline
\end{tabular}


De acuerdo con la Tabla 4, los mayores porcentajes se ubicaron en las categorías de Muy Bajo con un 26.5\%, Bajo con un $20.6 \%$ y Casi Bajo con un 14.7; mientras que en los niveles altos, sus frecuencias fueron más bajas, siendo la puntuación del nivel de Muy Alto de 14,7\%, indicando mayor inadaptación escolar en los adolescentes que no practican actividad física. Así mismo, los que practican actividad física, presentan bajos niveles de inadaptación escolar con puntuaciones como 32.4\% en Muy Bajo, 26.5\% en Bajo y 23.5\% en Casi Bajo, siendo relevante que los que practican actividad física tienen mayor motivación para la adaptación escolar.

Tabla 4

Frecuencias y Porcentajes de la Subescala TAMAl en los grupos de estudio en Inadaptación Escolar

\begin{tabular}{|c|c|c|c|c|c|c|c|}
\hline $\begin{array}{l}\text { Inadap. Escolar } \\
\text { No Actividad } \\
\text { Física }\end{array}$ & Categoría & Frecuencia & $\%$ & $\begin{array}{l}\text { Inadap. Escolar } \\
\text { Actividad } \\
\text { Física }\end{array}$ & Categoría & Frecuencia & $\%$ \\
\hline & Muy bajo & 9 & 26.5 & & Muy bajo & 11 & 32.4 \\
\hline & Bajo & 7 & 20.6 & & Bajo & 9 & 26.5 \\
\hline & Casi Bajo & 5 & 14.7 & & Casi Bajo & 8 & 23.5 \\
\hline & Medio & 4 & 11.8 & & Medio & 4 & 11.8 \\
\hline & Casi Alto & 1 & 2.9 & & Casi Alto & 2 & 5.9 \\
\hline & Alto & 3 & 8.8 & & Alto & 0 & 0 \\
\hline & Muy Alto & 5 & 14.7 & & Muy Alto & 0 & 0 \\
\hline & Total & 34 & 100 & & Total & 34 & 100 \\
\hline
\end{tabular}

De acuerdo a la Tabla 5 son altos los porcentajes en los niveles Muy Alto con un $38.2 \%$ y Alto con un $26.5 \%$ en la inadaptación social de la muestra que no practica actividad física; mientras que para el nivel Muy Bajo hay un 5,9\%; por su parte en los niveles Medio y Casi Alto hay un $38.2 \%$ y $34.4 \%$ respectivamente, en los sujetos que practican actividad física.

Tabla 5

Frecuencias y Porcentajes de la Subescala TAMAI en los grupos de estudio en Inadaptación Social

\begin{tabular}{|c|c|c|c|c|c|c|c|}
\hline $\begin{array}{l}\text { Inadap. Social } \\
\text { No Actividad } \\
\text { Física }\end{array}$ & Categoría & Frecuencia & $\%$ & $\begin{array}{c}\text { Inadap. Social } \\
\text { Actividad } \\
\text { Física }\end{array}$ & Categoría & Frecuencia & $\%$ \\
\hline & Muy bajo & 2 & 5.9 & & Muy bajo & 0 & 0 \\
\hline & Bajo & 0 & 0 & & Bajo & 0 & 0 \\
\hline & Casi Bajo & 0 & 0 & & Casi Bajo & 2 & 5.9 \\
\hline & Medio & 6 & 17.6 & & Medio & 13 & 38.2 \\
\hline & Casi Alto & 4 & 11.8 & & Casi Alto & 11 & 34.4 \\
\hline & Alto & 9 & 26.5 & & Alto & 4 & 11.8 \\
\hline & Muy Alto & 13 & 38.2 & & Muy Alto & 4 & 11.8 \\
\hline & Total & 34 & 100 & & Total & 34 & 100 \\
\hline
\end{tabular}


La Tabla 6 indica en los niveles Medio, con un 55.9\% y Muy Alto, con un $26.5 \%$, mayores frecuencias en insatisfacción familiar en relación con los niveles Casi Bajo, Bajo y Muy bajo en los que no practican actividad física, en los cuales no hubo respuesta; situación muy similar con las puntuaciones con los adolescentes que practican actividad física, debido a que en los niveles Medio, con un 64.7\% y Muy Alto, con un 20.6\%, fueron los que tuvieron mayores frecuencias.

Tabla 6

Frecuencias y Porcentajes de la Subescala TAMAl en los grupos de estudio: Insatisfacción Familiar

\begin{tabular}{|c|c|c|c|c|c|c|c|}
\hline $\begin{array}{c}\text { Insatisf } \\
\text { Familiar No } \\
\text { Actividad Física }\end{array}$ & Categoría & Frecuen. & $\%$ & $\begin{array}{c}\text { Insatisf. } \\
\text { Familiar } \\
\text { Actividad Física }\end{array}$ & Categoría & Frecuen. & $\%$ \\
\hline & Muy bajo & 0 & 0 & & Muy bajo & 0 & 0 \\
\hline & Bajo & 0 & 0 & & Bajo & 0 & 0 \\
\hline & Casi Bajo & 0 & 0 & & Casi Bajo & 0 & 0 \\
\hline & Medio & 19 & 55.9 & & Medio & 22 & 64.7 \\
\hline & Casi Alto & 6 & 17.6 & & Casi Alto & 5 & 14.7 \\
\hline & Alto & 0 & 0 & & Alto & 0 & 0 \\
\hline & Muy Alto & 9 & 26.5 & & Muy Alto & 7 & 20.6 \\
\hline & Total & 34 & 100 & & Total & 34 & 100 \\
\hline
\end{tabular}

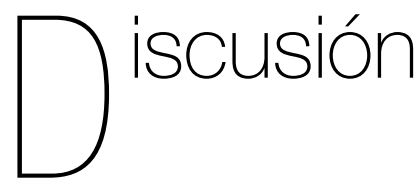

De acuerdo con el objetivo de la presente Investigación, el cual pretendió describir y comparar la adaptación en adolescentes que practican y que no practican actividad física, se puede concluir que se presenta inadaptación personal en los adolescentes que no practican actividad física, lo que podría estar asociado con la falta de motivación por parte del sujeto (Almeida, Salgado, \& Nogueira, 2011); propios de su etapa de desarrollo (Oliva, 2007) que se caracterizan por un auto-desajuste que no acepta la realidad que vive el adolescente, y que se manifiesta con comportamientos de evitación y escape, y con pensamientos negativos o catastróficos que impiden la realización de dichas actividades.

Por otra parte, Torregrosa, Belando y Moreno (2013) evidencian como con instrucciones controladoras a los adolescentes se reducen su motivación, siendo la actividad física la puesta en marcha de acciones positivas para su práctica y mantenimiento tales como horario, día de la práctica y conciliación de horarios; a esto se suman los hallazgos de Peiró, Valenciano, Beltrán y Devís (2014) quienes consideran que de acuerdo a la edad, hay días específicos a la semana en donde los refuerzos percibidos mantienen más la conducta de la práctica de la actividad física, así como tiempos contados en minutos que se asocian a una mayor satisfacción de los resultados obtenidos con la práctica (Agüilar, Ortegón, 
Mur, Sánchez, García, García García, \& Sánchez, 2014).

Los resultados del análisis estadístico descriptivo muestran puntuaciones significativas, siendo relevante que la falta de práctica de actividad física presenta mayor riesgo de inadaptación escolar y social. Lo anterior corrobora lo hallado por Gálvez, Rodríguez y Velandrino (2007) donde la práctica de actividad física genera bienestar biopsicosocial en el sujeto debido a la existencia de reforzadores (tanto tangibles como sociales en la práctica de actividades físicas), que son los que mantienen una conducta en la práctica; y que la presencia de ansiedad, depresión, agresión y exclusión de su grupo de pares, pueda deberse a que el adolescente no practica actividad física, o es mínimo el esfuerzo para la realización de la actividad o los resultados no reciben el refuerzo aprobatorio por parte de personas significativas (Molinero, Martínez, Garatachea, Márquez, \& Márquez, 2010).

También se puede concluir que la inadaptación escolar puede ser debida a que la presencia de problemáticas de índole familiar no permiten un desarrollo en cuanto a lo académico, y a que las preferencias de los adolescentes se dirigen a otro tipo de actividades (Rodrigo et al., 2004; Rodrigo et al., 2006). Igualmente la inadaptación escolar puede estar asociada con la falta de apoyo familiar debido a que el adolescente al estar en etapa de estudio, la atención positiva familiar estaría relacionada tanto con el incremento de la autoestima como con las recompensas que producen los resultados académicos, lo que comportamentalmente se evidenciaría con habilidades sociales, autorregulación, reduciendo pensamientos como aislamiento mental y ensoñación y por ende un mayor compromiso con la actividad física (Martínez, Musitu, Amador, \& Monreal, 2012).

Díaz y Martín (2011), resaltan la importancia de la adaptación escolar en los adolescentes, teniendo en cuenta variables como el sexo, evidenciando mayor adaptación por parte de las adolescentes debido a procesos como expectativas de seguir estudiando, sentido de pertenencia a la escuela, relaciones funcionales con el profesorado; apoyando la relación entre adaptación escolar y práctica de actividad física expuesta por Martínez et al., (2012) y González y Otero (2005), siendo relevante para la disminución en conductas agresivas en los adolescentes.

En lo concerniente al área social de acuerdo a los resultados de la investigación, se corroboran los aportes de Romero, Luengo, Gómez y Sobral, (2002), debido a que el adolescente para ajustarse no sólo requiere de la práctica de actividades físicas, sino también la interacción con sus pares, la manifestación de conductas prosociales, son aspectos reforzantes que ayudan a la autoestima del adolescente, reduciendo pensamientos negativos, o conductas que conduzcan a la afectación de su integridad (Oliva, Jiménez, Parra, \& Sánchez, 2008). Aún cuando en los resultados no se encontraron diferencias significativas en la insatisfacción familiar entre los que practican y no practican actividad física, si es fundamental resaltar el papel del apoyo familiar para el ajuste social del adolescente, lo que va en línea con las investigaciones que destacan la importancia de la familia en el mantenimiento de una práctica de actividad física (Pons, 
Queralt, Mars, García, \& Balaguer, 2010). Por su parte Rueger, Malecki y Demaray (2008), señalan que la calidad de la relación familiar contribuye a la reducción de agresividad en el caso de las mujeres, mientras que para los hombres el apoyo familiar mejoraría sus habilidades sociales y de liderazgo, lo que conlleva a una adaptación del adolescente, evitando sintomatologías de depresión o somatización.

De acuerdo con los resultados frente a la inadaptación personal, hay puntuaciones similares con las categorías Casi Alto y Casi Bajo, en los adolescentes que no practican actividad física; de la misma forma, puntuaciones similares en los que practican actividad física, siendo resultados que deberían profundizarse en próximas investigaciones. Esto se podría explicar según lo planteado por Molinero, Martínez, Garatachea y Márquez (2010), quienes refieren que cuando las actividades físicas no requieren mayor esfuerzo, no son atractivas al grupo de pares, y por lo tanto, no tendrían un valor mayor de refuerzo que lo podrían tener actividades de mayor esfuerzo y con recompensas tangibles y sociales inmediatas. Así mismo sería importante sensibilizar a los adolescentes sobre la práctica de la actividad física como un recurso para la prevención del sobrepeso y la obesidad (Aguilar et al., 2014).

Igualmente, el hecho que no existan diferencias estadísticamente significativas en cuanto a la adaptación personal del adolescente frente a la práctica o no de actividad física, corrobora lo hallado por Rodrigo et al., (2004), en donde asumir conductas de riesgo, está vinculada con experiencias que tienen propósitos como recibir la aceptación de su grupo de pares, especialmente en lo que respecta a relaciones interpersonales (Sánchez, Quiroga, Bragado, \& Martín, 2004).

Otros resultados significativos son los evidenciados en inadaptación social en los sujetos que no practican actividad física, lo que indicaría la necesidad de que en estos adolescentes se tuviera en cuenta el fortalecimiento del nivel educativo, la automotivación, la autoeficacia, el apoyo dado por otra persona significativa de su entorno, el tiempo disponible por parte del sujeto y la influencia del grupo de pares, dado que son factores que contribuirían a incrementar comportamientos relacionados con la práctica de la actividad física (Gálvez et al., 2005).

Los resultados obtenidos frente a la inadaptación escolar y social apoyan lo encontrado por Tapia et al., (2007), en el sentido que si el adolescente fortalece sus relaciones con su grupo de pares a través de conductas prosociales, recibiendo reforzadores que mantengan dichas conductas, como la aprobación y satisfacción de la otra persona y si el proceso de empatía es recíproco, el adolescente tendría menos posibilidades de manifestar baja laboriosidad en el aprendizaje, tener baja motivación por el estudio o manifestar conductas disruptivas en el aula, así mismo se podría presentar menor rechazo hacia los docentes o hacia las mismas instituciones escolares.

Las conclusiones de la investigación conducen a que la adaptación del adolescente debe ser holística, comprendiendo varias áreas de desarrollo para que el ajuste sea coherente con las demandas ambientales. Sí bien los resultados fueron enfáticos en lo escolar y 
social, se corroboran los hallazgos del TAMAI (Hernández, 1998), haciendo énfasis en la fuerte asociación entre estas dos áreas, debido a que de una alta motivación para el aprendizaje y de unos refuerzos a cambio como altas calificaciones, aceptación del grupo de pares, empatía con docentes, conducen a que aumente el autocontrol social, incrementar las relaciones sociales, pacifismo y comunicación asertiva.

La implementación de la actividad física en la adolescencia, comenzando desde la infancia, aumenta la posibilidad de ajuste en esta etapa de desarrollo, reduciendo comportamientos agresivos y aumentando la probabilidad de aparición y mantenimiento de conducta prosocial. Por otra parte, la implementación de actividad física en las organizaciones debe tener un protocolo, objetivos y propósitos que capten la atención de los adolescentes, puesto que de acuerdo con los hallazgos de Gálvez et al., (2005), cuando existe dicha programación, sumado a contar con instalaciones físicas y herramientas adecuadas, aumenta la motivación por parte del adolescente, incluyendo el apoyo por parte de las personas significativas de su entorno.

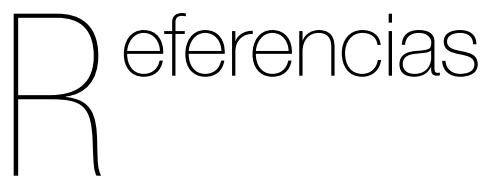

Aguilar, M., Ortegón, A., Mur, N., Sánchez, J., García, J., García, I., \& Sánchez, A. (2014). Programas de actividad física para reducir sobrepeso y obesidad en niños y adolescentes; revisión sistemática. Nutrición Hospitalaria, 30(4), 727-740.
Almeida, C., Salgado, J., \& Nogueira, D. (2011). Estilo de vida y el peso corporal en una comunidad portuguesa en transición: un estudio de la relación entre la actividad física, los hábitos alimentarios y el índice de masa corporal. Revista Iberoamericana de Psicología y Salud, 2(1), 27-56.

Ángeles, M. (2011). Factores de riesgo y factores de protección en la adolescencia: análisis de contenido a través de grupos de discusión. Terapia Psicológica, 29(1), 85-95.

Avellaneda, A., Gras, M., \& Font, S. (2010). Patrones de consumo de alcohol en la adolescencia. Psicothema, 22(2), 189-195.

Booth, M. (2000). Assessment of physical activity: an international perspective. Research Quarterly for Exercise and Sport, 71(2), 114-20.

Carrasco, D., Gómez, E., \& Staforelli, A. (2009). Obesidad y adolescencia: exploración de aspectos relacionales y emocionales. Terapia Psicológica, 7(1), 143-149.

Cetina, C., \& Moreno, J. (2013). Adaptación en adolescentes que practican actividad física frente a los que no practican. (Trabajo de grado para optar al título de Especialista en Psicología Clínica de la niñez y de la Adolescencia). Chía. Universidad de La Sabana. Recuperado de: http://hdl.handle.net/10818/9069

De la Fuente, J., Peralta, F., \& Sánchez, M. (2009). Autorregulación personal y percepción de los comportamientos escolares desadaptativos. Psicothema, 21(4), 548-554. 
Díaz, M. (2005). La violencia entre iguales en la adolescencia y su prevención desde la escuela. Psicothema, 17(4), 549-558.

Díaz, M., \& Martín, G. (2011). Convivencia y aprendizaje escolar en la adolescencia desde una perspectiva de género. Psicothema, 23(2), 252-259.

Gálvez, A., Rodríguez, P., \& Velandrino, A. (2007). Influencia de determinados motivos de práctica físico-deportiva sobre los niveles de actividad física habitual en adolescentes. Cuadernos de Psicología del Deporte, 7(1), 71-84.

Giraldo, D., Poveda, E., Forero, Y., Mendivil, C., \& Castro, L. (2008). Actividad física autorreportada, comparación con indicadores antropométricos de grasa corporal en un grupo de escolares de Bogotá y de cinco departamentos del centro-oriente. Biomédica, 28(3), 386-95.

Gómez, Y. (2007). Cognición, emoción y sintomatología depresiva en adolescentes escolarizados. Revista Latinoamericana de Psicología, 39(3), 435-447

González, Á., \& Otero, M. (2005). Actitudes de los padres ante la promoción de la actividad física y deportiva de las chicas en edad escolar. Cuadernos de Psicología del Deporte, 5(1, 2), 173-195.

Hernández, P. (1998). Test Autoevaluativo Multifactorial de Adaptación Infantil: TAMAI.Madrid: TEA.

Hernández, R., Fernández, C., \& Baptista, P. (2010). Metodología de la Investigación (4 edición). Bogotá: McGraw Hill.

Lillo, J. (2004). Crecimiento y comportamiento en la Adolescencia. Revista de la
Asociación Española de Neuropsiquiatría, 90, 57-71.

Martínez, B., Musitu, G., Amador, L., \& Monreal, M. (2012) Estatus sociométrico y violencia escolar en adolescentes: implicaciones de la autoestima, la familia y la escuela. Revista Latinoamericana de Psicología, 44(2), 55-66.

Molina, J., Sandín, B., \& Chorot, P. (2014). Sensibilidad a la ansiedad y presión psicológica: efectos sobre el rendimiento deportivo en adolescentes. Cuadernos de Psicología del Deporte, 14(1), 45-54.

Molinero, O., Martínez, R., Garatachea, N., Márquez, S., \& Márquez, S. (2010). Pautas de actividad física de adolescentes españolas: diferencias mediadas por la participación deportiva y el día de la semana. Revista de Psicología del Deporte, 19(1), 103-116.

Oliva, A. (2007). Desarrollo cerebral y asunción de riesgos durante la adolescencia. Apuntes de Psicología, 25(3), 239-254.

Oliva, A., Jiménez, J., Parra, A., \& Sánchez, I. (2008). Acontecimientos vitales estresantes, resiliencia y ajuste adolescente. Revista de Psicopatología y Psicología Clínica, 13(1), 53-62.

Parada, A., Klaasen, J., Lisboa, C., Saldías, F., Mendoza, L., \& Díaz, O. (2011). Reducción de la actividad física en pacientes con enfermedad pulmonar obstructiva crónica. Revista Médica de Chile, 139(12), 1562-1572.

Pastor, Y., Balaguer, I., \& García, M. (2005). Relaciones entre el autoconcepto y el estilo de vida saludable en la adolescencia 
media: un modelo exploratorio. Psicothema, 18(1), 18-24.

Peiró, C., Valenciano, J., Beltrán, V., \& Devís, J. (2014). Variabilidad de la actividad física en adolescentes españoles de 1718 años en función del tipo de jornada y época del año. Revista de Psicología del Deporte, 23(2), 347-354.

Pons, D., Queralt, A., Mars, L., García, M., Balaguer, I., \& Balaguer, I. (2010). Estudio cualitativo de las conductas de salud en la primera adolescencia. Revista Latinoamericana de Psicología, 42(2), 237-250.

Rodrigo, M., Máiquez, M., García, M., Medina, A., Martínez, M., \& Martín, J. (2006). La influencia de las características personales y contextuales en los estilos de vida en la adolescencia: aplicaciones para la intervención en contextos de riesgo psicosocial. Anuario de Psicología, 37(3), 259-276.

Rodrigo, M., Máiquez, M., García, M., Mendoza, R., Rubio, A., Martínez, A., \& Martín J. (2004). Relaciones padreshijos y estilos de vida en la adolescencia. Psicothema, 16(2), 203-210.

Romero, E., Luengo, A., Gómez, J., \& Sobral, J. (2002). La estructura de los rasgos de personalidad en adolescentes: El Modelo de Cinco Factores y los Cinco Alternativos. Psicothema, 14(1), 134-143.

Rueger, S., Malecki, C., \& Demaray, M. (2008). Gender Differences in the Relationship Between Perceived Social Support and Student Adjustment During Early Adolescence. School Psychology Quarterly, 23(4), 496-514.
Sánchez-Bernardos, M., Quiroga, M., Bragado, M., \& Martín, S. (2004). Autodiscrepancias y relaciones interpersonales en la adolescencia. Psicothema, 16(4), 582-586.

Seder, A., Villalonga, H., \& Domingo, C. (2014). Estudio del rendimiento académico en atletas adolescentes del club de atletismo playas de Castellón. Revista Ciencias del Deporte, 10(1), 53-66.

Tapia, N., Castro, R., \& Monestel, N. (2007). El desarrollo sociomoral de adolescentes de Costa Rica según el modelo de gibbs. Revista Latinoamericana de Psicología, 39(3), 449-471.

Torregrosa, D., Belando, N., \& Moreno, J. (2013). Predicción de la satisfacción con la vida en practicantes de ejercicio físico saludable. Cuadernos de Psicología del Deporte, 14(1), 117-122.

Urdampilleta, A., González, J., Infante, G., \& Goñi, A. (2011). La Tensión arterial sistémica y el autoconcepto físico en la adolescencia. Cuadernos de Psicología del Deporte, 11(2), 25-33.

Varela, M., Duarte, C., Salazar, I., Lema, L., \& Tamayo, J. (2011). Actividad física y sedentarismo en jóvenes universitarios de Colombia: prácticas, motivos y recursos para realizarlas. Colombia Médica, 42(3), 269-77. 